\title{
Pediatric vision screening: The Colorado school nurse experience
}

\author{
Rebecca S. Braverman*1,2 \\ ${ }^{1}$ Department of Ophthalmology, School of Medicine, University of Colorado, Aurora, CO, United States \\ ${ }^{2}$ Childrens Hospital Colorado, Aurora CO, United States
}

Received: February 24, 2018

Accepted: May 13, 2018

Online Published: June 25, 2018

DOI: $10.5430 /$ jnep.v8n11p47

URL: https://doi.org/10.5430/jnep.v8n11p47

\begin{abstract}
Colorado schools are required by law to perform vision screening. The aim of the study was to elucidate the vision screening process from the school nurse perspective. An electronic questionnaire distributed to Colorado school nurses, and developed by the author, was used to query Colorado nurses about the logistical challenges they face when performing vision screening. Common challenges faced when performing vision screening included: inadequate staff to perform vision screening, inadequate space to complete testing, uncooperative students, and poor parental compliance with referrals for further evaluation after their child failed their vision screen. Most nurses do not use instrument-based screening. Many nurses did not properly occlude the eyes when performing monocular visual acuity testing, which may lead to false negative screening results. The results of the questionnaire suggest there are opportunities to improve the vision screening process by nurses in Colorado schools.
\end{abstract}

Key Words: Vision screening, Instrument-based vision screening, Amblyopia

\section{INTRODUCTION}

Vision screening can identify children at risk for permanent vision loss. The US Preventive Services Task Force recommends vision screening at least once in all children aged 3 to 5 years to detect amblyopia or its risk factors. ${ }^{[1]}$ Nurses refer children who fail their vision screening for further evaluation and management by an optometrist or ophthalmologist. Most states in the US have state laws that require vision screening in schools. ${ }^{[2]}$ Although states mandate vision screening in schools, little information is available that details the logistical challenges school nurses face when tasked with providing vision screening services for their students. The author's home state of Colorado requires vision screening in grades K-3, 5, 7, 9 and referral for failed screening. Vision screening and referral guidelines are provided by the
Colorado Department of Education. ${ }^{[3]}$ The aim of this short report is to document the challenges Colorado school nurses face when performing vision screening.

\section{MethodS}

The Colorado Multiple Institutional Review Board evaluated the study and approved it prior to the author sending a vision screening experience questionnaire electronically to Colorado school nurses. The questionnaire developed by the author was sent electronically to members of the Colorado Department of Education School Nurse listserv. The questionnaire aimed to identify the characteristics of the respondents, and the challenges they face when tasked with providing school vision screening services. Members of the listserv were Colorado school nurses. Nurses were employed

\footnotetext{
* Correspondence: Rebecca S. Braverman; Email: Rebecca.SandsBraverman@ucdenver.edu; Address: Department of Ophthalmology, School of Medicine, University of Colorado, Aurora, CO, United States.
} 
by both private and public schools in urban and rural settings. The questionnaire had twenty-six questions. The questions were multiple choice and short answer. Statistical analysis was done by the author.

\section{Results}

The questionnaire response rate was $28 \%$ (218 of 774). Most respondents worked in public schools $(88 \%)$. Respondents worked in urban schools $(72 \%)$ more than rural or frontier schools. They were responsible for several hundred to several thousand children. Nurses learned how to perform vision screening at the district level, state level, by self-study and by miscellaneous means.

Nurses reported many logistic challenges when tasked with providing vision screening (see Figure 1).

Inadequate staff and space for screening were the most common complaints. Volunteer assistance with screening was greatly appreciated. However, some respondents voiced concerns about volunteer training. Lack of space to perform screening led to a "chaotic" environment. Reported screening locations included hallways and cafeterias. Lighting conditions were often too dim or too bright.

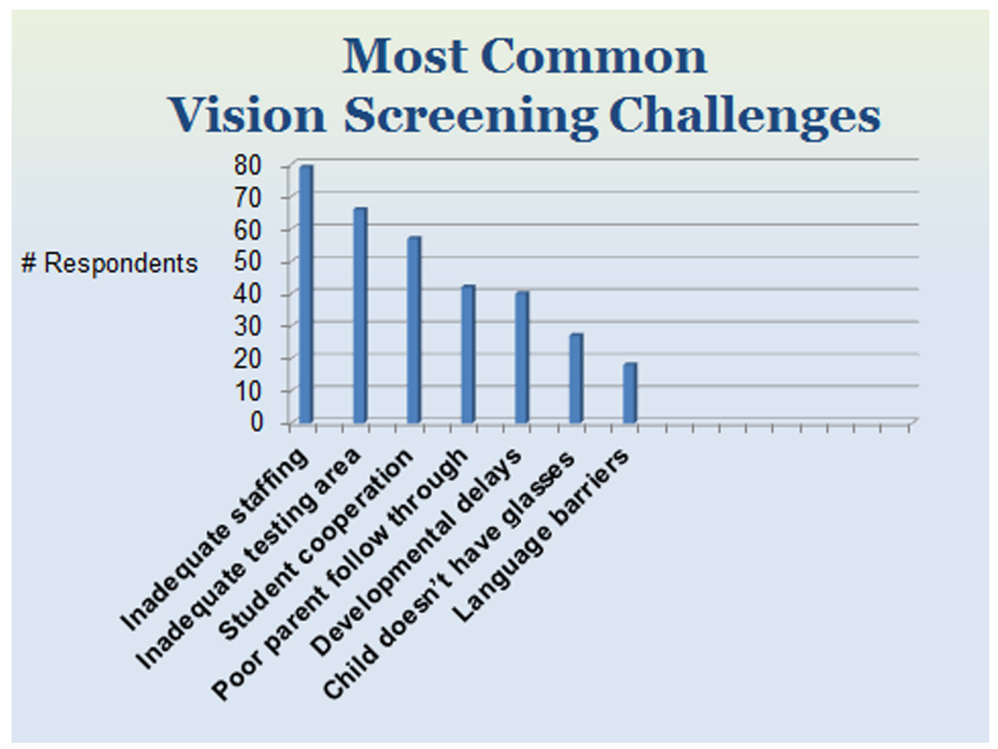

Figure 1. Most common vision screening challenges

Children did not cooperate with vision screening for a number of reasons. Young children were fearful and had trouble following directions. Some children "faked" responses because they wanted glasses. Other children did not like their glasses and would perform screening without them. Certain older children chose not to cooperate. Nurses found it particularly difficult to screen children who were young, had autism, developmental delays or language barriers.

Scheduling screening was difficult. Nurses found it tough to schedule screenings and limit the time children were out of the classroom. Children absent on screening days were hard to reschedule. Some teachers did not "buy in" to the process and failed to get students to the screening location on time.

Nurses told families about screening failures with written letters, phone calls and emails. Children who failed their screening were referred to optometrists $(41 \%)$, ophthalmologists (30\%), parents' choice (22\%) and other (7\%) for further evaluation. Nurses contacted parents by phone or email if they did not get referral results. If they were unable to contact the parents, some respondents spoke with teachers or repeated the screening. Many felt they did not have enough time to track down referral results. Noncompliance with referrals was reported to be common.

School nurses had difficulties communicating with eye doctors and tracking students who received eye care prior to screening. Only one third of respondents got a letter from the eye doctor after a referral was complete. Poor communication lead to uncertainty about a child's need for glasses or treatment. Lack of feedback about referrals made it difficult for nurses to determine if their screening program was over or under referring.

Most nurses did not use instrument based screening and did not have money to purchase the equipment. However, some young students received instrument screening by Lions Club volunteers. Nurses reported increased success using instruments to screen young children. 
Nurses followed Colorado guidelines for vision screening and referrals. However, less than $1 \%$ used adhesive patches to ensure monocular acuity testing. Seventy-eight percent of respondents allowed children to occlude their own eyes during monocular vision testing.

\section{Discussion}

Colorado state law requires schools to perform vision screening. Nurses responsible for vision screening programs have many challenges. They do not have uniform testing environments, uniform training or sufficient staff. Fortunately, there are a few things nurses could do to improve the screening process. Nurses could increase their use of instrument-based screening for certain children. Instrument based screening is useful for young children, those with language barriers or developmental delays. ${ }^{[4]}$ Children with medical conditions that increase the risk of eye disease could be referred to an eye doctor without being screened. ${ }^{[5]}$ Students who receive eye care could be exempt from screening.

Vision screening accuracy may be improved by using adhesive eye patches. A child with poor vision in one eye may peek around the occluder during monocular vision testing. Peeking could trick the examiner into believing the child has normal vision. Examiners should use adhesive patches to prevent peeking. The author has coined the phrase "patching prevents peeking" and uses the phrase when training screeners how to check the vision one eye at a time.

Noncompliance with eye care referrals is a big problem. Caregivers fail to comply with referrals because of communication problems, language barriers and denial. ${ }^{[6]}$ Nurses spend a lot of time communicating with families when a referral is necessary. Perhaps education as well as communication is needed. Parents who understand their child is at risk for lifelong vision loss are more likely to comply with the referral.
Eye care providers can help school nurses in several ways. They can accept referrals from nurses for failed vision screening. Physicians can improve communication with nurses by sending a letter with exam findings. Finally, they can share their knowledge and teach nurses about pediatric eye disease and vision screening.

The questionnaire format of the study is a limitation. However, the questionnaire format was selected because of it was easy to distribute it across a large geographic area and incurred little cost. An additional study limitation is the low response rate which may induce bias. Further bias may be induced by the under-representation of private school nurses in the study. Most respondents were public school nurses, and the experience of private school nurses may be much different.

\section{Conclusions}

School vision screening programs can identify children with sight threatening conditions. School nurses responsible for vision screening programs must overcome many obstacles to perform mass screenings. Fortunately, there are opportunities for improvement. Increased use of instrument based screening for certain children could increase screening success rates. Children with medical conditions that increase the risk of eye disease, or prevent cooperation, could be referred for eye care without being screened. Students who receive eye care could be exempt from screening. Use of adhesive patches during monocular vision testing could increase the accuracy of screening results. Eye care providers can support nurses by seeing their referrals and improving communication.

\section{CONFLicts OF INTEREST Disclosure}

The author has a financial relationship with Welch Allyn as a consultant that does influence this report.

\section{REFERENCES}

[1] US Preventive Services Task Force, Grossman DC, Curry SJ, et al. Vision Screening in Children Aged 6 Months to 5 Years: US Preventive Services Task ForceRecommendation Statement. JAMA. 2017 Sep 5; 318(9): 836-844.

[2] American Association for Pediatric Ophthalmology and Strabismus. State-by- State Vision Screening Requirements. Available from: https://www . aapos.org/resources/state_by_state _vision_screening_requirement/

[3] Colorado Department of Education 2015 Guidelines for School Vision Screening Programs: Kindergarten Through Grade 12. Available from: https://www. Colorado.state.co.us/healt handwellness/guidelinesforschoolvisionscreeningpro gramsk-122016pdf
[4] Nottingham Chaplin PK, Baldonado K, Hutchinson A, Moore B. Vision and eye health: moving into the digital age with instrumentbased vision screening. NASN Sch Nurse. 2015; 30(3): 154-160. PMid:25870098 https://doi .org/10.1177/1942602X155810 54

[5] Cotter SA, Cyert LA, Miller JM, et al. Vision screening for children 36 to < 72 months: recommended practices. Optom Vis Sci. 2015; 92(1): 6-16. PMid:25562476 https://doi.org/10.1097/OPX. 0000000000000429

[6] Tjiam AM, Groenewoud JH, Passchier J, et al. Determinants and outcome of unsuccessful referral after positive screening in a large birth-cohort study of population-based vision screening. J AAPOS 2011; 15(3): 256-262. PMid:21777799 https://doi.org/10.1 016/j.jaapos.2011.01.159 